\title{
Mechanical performance and in vivo bioactivity of functionally graded PEEK-HA biocomposite materials
}

\author{
Rui Ma $\cdot$ Lin Fang $\cdot$ Zhongkuan Luo $\cdot$ \\ Luqian Weng $\cdot$ Shenhua Song $\cdot$ Ruisheng Zheng • \\ Hongyuan Sun $\cdot$ Huide Fu
}

Received: 4 September 2013/Accepted: 20 January 2014/Published online: 30 January 2014

(C) The Author(s) 2014. This article is published with open access at Springerlink.com

\begin{abstract}
A functionally graded material (FGM) of polyetheretherketone (PEEK)-hydroxyapatite (HA) with a symmetrical three-layer structure containing 8.7, 2.6 and $8.7 \mathrm{vol} \% \mathrm{HA}$, respectively, is successfully fabricated by hot pressing and evaluated by scanning electron microscopy and tensile testing. A good bonding between the HA particles and the PEEK matrix is achieved by virtue of an in situ method in fabricating each PEEK-HA composite layer. There are no apparent microcracks and microscopic interfaces within each layer of the FGM. The symmetrical PEEK-HA FGM does not fall apart or delaminate during loading with a tensile strength of $86.2 \mathrm{MPa}$, and its fracture energy is $56.5 \mathrm{~kJ} / \mathrm{m}^{2}$. Furthermore, in vivo bioactivity of the FGM sample is also evaluated, showing that it has sound biocompability and bioactivity. Accordingly, the FGM in the future may be a promising biomaterial to be used as replacement implant of human bone through a proper design to balance mechanical properties and bioactivity.
\end{abstract}

Keywords Functionally graded material - Biomaterials . Composites materials $\cdot$ Bioactivity $\cdot$ Hydroxyapatite

R. Ma $\cdot$ R. Zheng

Key Laboratory of Optoelectronic Devices and Systems of Ministry of Education and Guangdong Province, College of Optoelectronic Engineering, Shenzhen University,

Shenzhen 518060, China

R. Ma $\cdot$ L. Fang $(\bowtie) \cdot$ Z. Luo $\cdot$ H. Sun $\cdot$ H. Fu

College of Chemistry and Chemical Engineering, Shenzhen

University, Shenzhen 518060, China

e-mail: fanglinhit@163.com

L. Weng $\cdot$ S. Song

Materials Science and Engineering, Shenzhen Graduate School, Harbin Institute of Technology, Shenzhen 518055, China

\section{Introduction}

Polymeric composite biomaterials, as implant materials for replacing hard tissues of human body, have attracted increasing attention due to their designed structure and physical properties overcoming the disadvantages of metallic and ceramic materials currently adopted in orthopedic application [1-3]. The polymeric composite materials prepared by the addition of bioactive hydroxyapatite (HA) fillers in the polyethylene (PE) matrix, as bone analogue, were first introduced by Bonfield [4]. Bakar et al.' [5] study exhibited that the mechanical strength of polyetheretherketone (PEEK)-HA composite materials can be increased substantially, as compared with PE-HA composites, due to the PEEK excellent mechanical performance. However, incorporating a larger amount of HA fillers into the PEEK matrix necessary for achieving good bioactivity generally results in the brittleness of the composite materials accompanied with a significant decrease in fracture energy [6, 7], which could limit their applications as bone implants since the composite materials without desirable ductility are not safe for use in the consideration of avoiding the catastrophic failure of implants. The functionally graded PEEK-HA composite materials with an outer layer containing high HA content and inner layer containing lower HA contents could overcome these problems and ensure both their outstanding mechanical properties and excellent biological properties. In fact, functionally graded materials (FGMs) for biological applications have been investigated for years. Mishina et al. [8] studied $\mathrm{ZrO}_{2} / \mathrm{AlSi} 316 \mathrm{~L}$ FGMs for joint prostheses applications to increase the fracture toughness of ceramics and avoid the degradation and toxicity of metals. Pei et al. [9] and Rabiei et al. [10] have successfully fabricated the functionally graded carbon nanotubes/HA composite 
coatings and pure HA coatings on titanium alloys, respectively, to improve the bioactivity of the metallic materials. However, the interfacial binding of different materials must be greatly improved before they can be practically used. The functionally graded PEEK-HA composite materials could overcome the weakness of those materials since the matrix material of the different layers in the functionally graded PEEK-HA composites is the PEEK, but up to date no investigation has been reported on this field. In the present study, the functionally graded PEEK-HA composite materials with a three-layer structure were fabricated by hot pressing. The PEEK-2.6 vol\% HA composite was chosen as the inner layer due to its optimal mechanical properties [11], while the PEEK-8.7 vol\% HA composite was used as the outer layer to enhance the materials bioactivity. The structure and mechanical properties of FGM of PEEK-HA samples are characterized, and the bioactivity of the FGM with bone tissues in the rats are systematically investigated by in vivo studies and discussed.

\section{Experimental}

Commercial HA powders (99.5\% purity) in the experiment are needle-like with a primary particle size about $200 \mathrm{~nm}$ long and $50 \mathrm{~nm}$ wide, and with $92 \%$ crystallinity. The PEEK-HA composite samples were respectively prepared by an in situ synthesis process as follows. A certain amount of di-terbutyl peroxide, $p$-dihydroxybenzene, sulfobenzide, $\mathrm{K}_{2} \mathrm{CO}_{3}$ and $\mathrm{Na}_{2} \mathrm{CO}_{3}$ with a mass ratio of 1:2:5:0.2:0.4 were charged in a $500 \mathrm{ml}$ four-necked flask equipped with a reflux condenser. The mixture was first stirred for $0.5 \mathrm{~h}$ at ambient temperature, subsequently heated to $320^{\circ} \mathrm{C}$ at $10{ }^{\circ} \mathrm{C} /$ min under constantly stirring in pure nitrogen atmosphere. At $320^{\circ} \mathrm{C}$, HA powder with different contents was added respectively, and the mixture was held for $2 \mathrm{~h}$ at $320{ }^{\circ} \mathrm{C}$ before cooling. The composite materials were harvested by washing the products with deionized water and acetone for three times respectively, and dried in vacuum at $120{ }^{\circ} \mathrm{C}$ for $48 \mathrm{~h}$. The synthesized HA/PEEK composites were burned in muffle at $700{ }^{\circ} \mathrm{C}$ for $2 \mathrm{~h}$, in which PEEK phase of the composites except HA phase are carbonized. And then, the burned products are filtered and washed to remove the carbonized PEEK phase. The final products are HA phase in composites. The HA content in the final composite products was $2.6 \mathrm{vol} \%$, 8.7 vol\% respectively, measured by the weight loss of the samples in burning process.

The FGM samples were fabricated by stacking PEEKHA composite powders containing 8.7, 2.6 and 8.7 vol\% HA, respectively, into three layers in a steel die. The layer thickness of the PEEK-2.6 vol\% HA composite is about twice the thickness of the PEEK-8.7 vol\% HA layer in the samples, and thickness of outer layer and inter layer are 1 and $2 \mathrm{~mm}$, respectively. The samples were heated from 25 to $340{ }^{\circ} \mathrm{C}$ at a rate of $20{ }^{\circ} \mathrm{C} / \mathrm{min}$ and held there for $10 \mathrm{mins}$ under a pressure of $50 \mathrm{MPa}$, then cooled down to $25^{\circ} \mathrm{C}$ at the same rate. The monolithic PEEK-HA composite samples with 2.6 and 8.7 vol\% HA, respectively, were prepared by the same process for comparison. The polished and fracture surfaces of the samples were observed using scanning electron microscopy (SEM) (Hitachi S-4700). The tensile strength tests were performed on an Instron (WDN-100) material testing system with a cross-head speed of $0.5 \mathrm{~mm} / \mathrm{min}$, The mini rectangular tensile specimen with a gauge size of $20 \mathrm{~mm} \times 4 \mathrm{~mm} \times 7 \mathrm{~mm}$ was used for the tensile experiment on the Instron.

Sprague-Dawley rats were used for biological tests, and the test protocols were strictly followed and the animals were cared for in adequate facilities that safeguarded their well-beings throughout the experiments. The implant samples with a $5 \times 3 \times 3 \mathrm{~mm}$ rectangle shape were sterilized by a steam autoclave at $120{ }^{\circ} \mathrm{C}$ for 30 mins. A total of 15 implants (5 each of autogenous bone, PEEK$2.6 \mathrm{vol} \% \mathrm{HA}$ and FGM) were randomly distributed. Under general anesthesia the implants were inserted into the fracture part of the rat femurs, and the wound was fixed and sutured. The rats were sacrificed after 1 and 3 months, and the implants together with surrounding tissues were obtained and pretreated in $4 \%$ formalin. Subsequently the tissues were separated from the implant, fixed with paraffin, and then stained with hematoxylin-eoxin for histological examination by optical microscope. Moreover, cross sectional area, maximum, minimum and average thickness of new born bone on the implants were respectively examined by Image-Pro Express $\mathrm{C}$ system. The results were also analyzed statistically by using SPSS 13.0 version for windows software.

\section{Results and discussion}

Figure 1 shows the SEM images of the polished and fracture surfaces of the FGM samples in the vicinity of the layer interface. The white spots in Fig. 1a are HA fillers embedded in the PEEK matrix, and the profile surface of the composite looks smooth, suggesting the presence of strong interaction at the HA-PEEK interface. In Fig. 1a, there was no hole left from the debonded HA fillers in the polishing, different from the report of Wang et al. [12]. The different layers, which can be identified by the HA contents changing from one side of the interface to the other side (Fig. 1a), are observed to be bound together without defects. Compared with others' researches [6, 13], it shows there were good dispersion and distribution of HA fillers in 
Fig. 1 SEM images of a polished surface and b fracture surface across the PEEK-HA FGM. c Energy spectrum analysis (a)

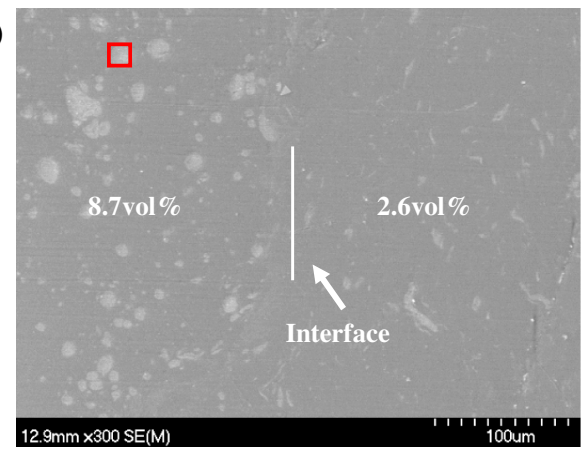

(b)

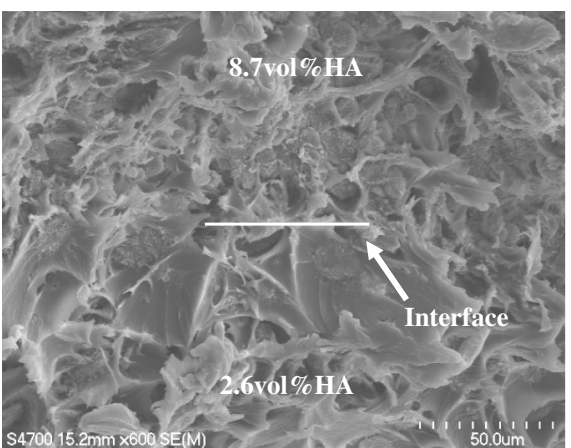

(c)
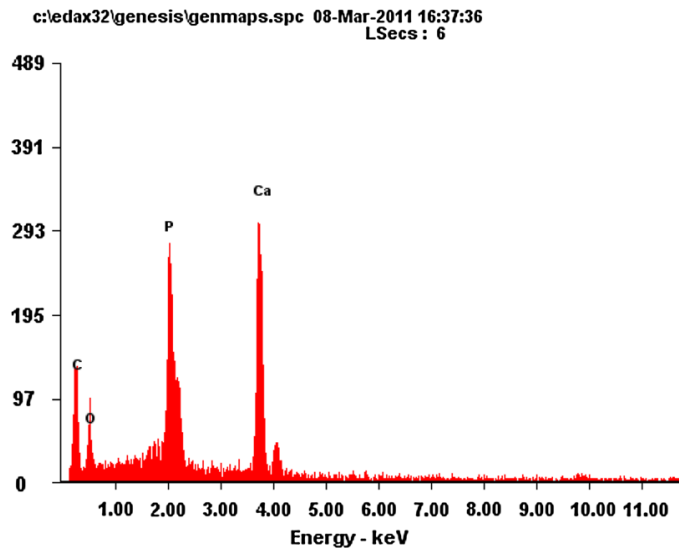

the PEEK matrix, and the HA particles are uniform in size, indicating that in situ polymerization of PEEK/HA would improve the adhesion of HA particles with the PEEK matrix, and avoid obvious aggregation of HA in the PEEK/ HA composites. The formation of the HA-PEEK strong bonding in the study is attributed to in situ synthesis process (see Fig. 2). In the synthesis process of the composite materials, HA particles were mixed into PEEK oligomers with short molecule chains and low viscosity, and good wetting and contacting between HA fillers and PEEK oligomers were achieved. With continuing polymerization, PEEK oligomers on HA surfaces became polymeric molecules with long molecular chains, which firmly wrapped on HA surface, similar to that of in situ preparation of carbonated $\mathrm{HA} /$ poly(4-vinyl pyridine-co-styrene) nano composite materials [14].

The fractograph of the sample (Fig. 1b) shows no delamination occurrence in tensile tests, indicating the strong cohesion between the layers. There are obviously different fractographs showed on the fracture surface in Fig. 1b, due to the different layers with the different HA contents. And the strong interfacial binding nature of the functionally graded PEEK-HA composite materials has great advantages over other functionally graded biomaterials for implant applications [15, 16]. From the EDX analysis of Fig. 1c, it is found that the ratio of $\mathrm{Ca} / \mathrm{P}$ is 1.681 in the HA (white spots, see Fig. 1a), which is close to human body (1.67).

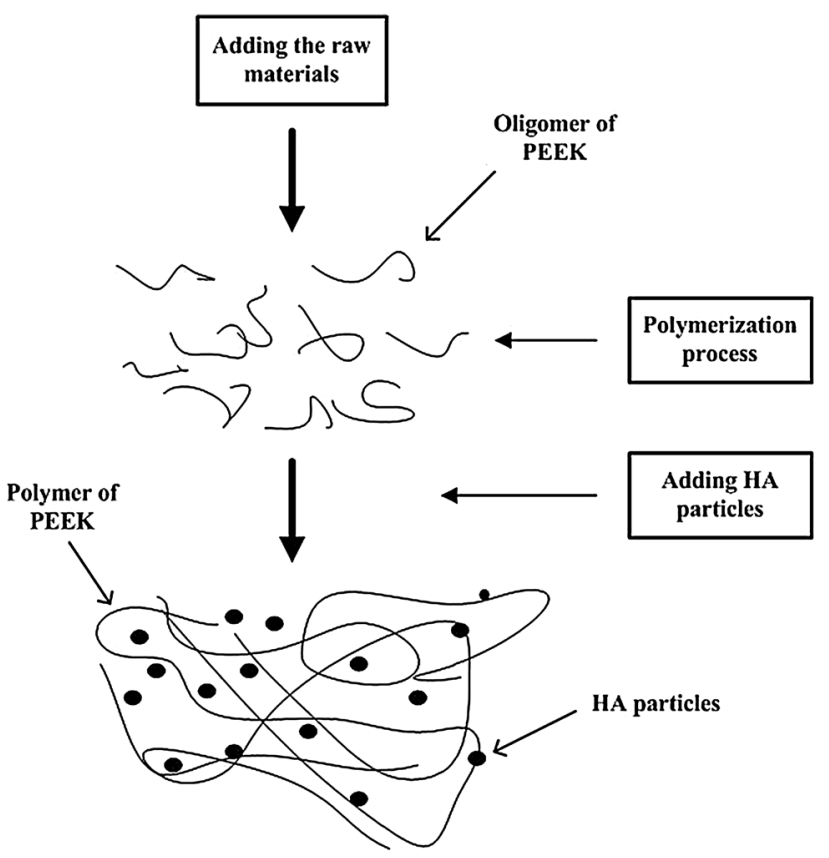

Fig. 2 Diagram of HA-PEEK composites by in situ method

The typical load-displacement curves for monolithic PEEK-2.6 vol\% HA samples and PEEK-8.7 vol\% HA samples as well as FGM samples are shown in Fig. 3. Their tensile strengths are 107, 75.4 and 86.2 MPa, respectively. It is found in Fig. 3 that E of $2.6 \mathrm{vol} \%$ and FGM samples 
are similar and lower than 8.7 vol\% one, which is consistent with our previous study [17], since the effective interface around HA particles have a significant effect on the composite elastic properties. As the HA particles size in composites is increased, the elastic properties increase due to the effect of interface on the composite. However, for further increases in HA particles size beyond some size, the ratio of the volume of the interface to the volume of the effective particle decreases to some extent, the effect of the effect interface on the composite elastic properties diminishes [18]. The fracture energies of the samples, estimated from the area under the load-placement curves, are about $88.9,10.8$ and $56.5 \mathrm{~kJ} / \mathrm{m}^{2}$, respectively. The displacement of the FGM sample and its fracture energy are 300 and $520 \%$ of those of the monolithic composite samples with $8.7 \mathrm{vol} \% \mathrm{HA}$, indicating that the generated fracture of the FGM is more difficult than the monolithic PEEK-8.7 vol\% HA composite.

As seen in Fig. 5, the monolithic PEEK-2.6 vol\% HA composite shows better tensile strength and ductility, as compared with the monolithic PEEK-8.7 vol\% HA composite. The previous studies [19] show that the local stress in composites materials under load can be more easily transferred into rigid particles with the addition of HA particles, resulting in a higher mechanical strength, when the particles are in intimate contact with the polymer matrix. Moreover, HA fillers in composites can interrupt and delay the propagation of micro-cracks or even stop their growth via stress distribution, which is also attributed to improve tensile strength [20].

The fractograph (Fig. 1b) shows a drawn-out fibration characteristic around HA fillers in the PEEK-8.7 vol\% HA layer of the FGM sample, suggesting that this layer has some plasticity. In contrast, the monolithic composite with

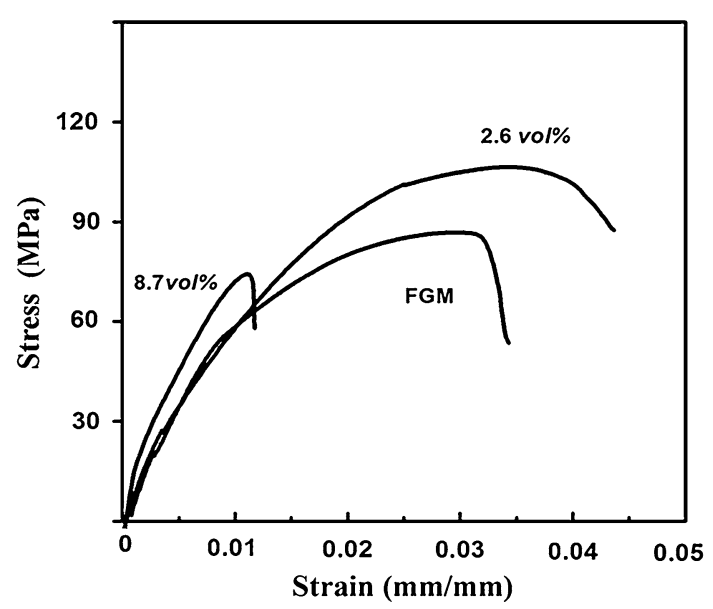

Fig. 3 Typical load-displacement curves of FGM and PEEK8.7 vol $\%$ HA composite with the one for PEEK-2.6 vol\% HA composite plotted for comparison
8.7 vol\% HA exhibits a brittle failure mode, in accordance with the tensile curve (Fig. 3) and the SEM observation that gives a relatively smooth fracture surface (Fig. 4b). The fractograph of the PEEK-2.6 vol\% HA layer in the FGM sample (Fig. 1b) shows the plastic nature with the stripes due to drawing-out effects, which is similar to the monolithic composite samples with 2.6 vol\% HA (Fig. 4a). Therefore, although the tensile strength of the FGM sample is about the average value of the monolithic PEEK2.6 vol\% HA and PEEK-8.7 vol\% HA composite samples, its ductility is substantially improved as compared with the monolithic PEEK-8.7 vol\% HA sample.

These results suggest that the mechanical properties of the functionally graded composites may be modified to a certain degree to meet various application requirements by optimizing the configuration design, such as each layer composition, and the thickness and number of layers.

After the samples implantation, the incision part of the rats recovered normally without infection, suggesting that samples had no toxicity to tissue and cells. Figure 5 gives the histological results of the body tissues in contact with the FGM, monolithic PEEK-2.6 vol\% HA composite as well as autogenous bone samples implanted in the rats for 1 and 3 months. In general, the bone repair in trauma can be characterized by the different stages from hematoma formation, granulation tissue formation, fibrous callus formation, and osseous callus formation to poroma recreation. In the monolithic PEEK-2.6 vol\% HA sample implanted for 1 month, the granulation tissues with inflammatory cells were dominant together with a small quantity of collagenic connection tissues (Fig. 5a), indicating that the bone repair after 1 month was still in the granulation tissue formation stage. And in the FGM sample implanted for 1 month a large quantity of collagenic connection tissues had been formed along with some osteoblasts besides osteocytes (Fig. 5c), indicating that the bone repair was in the stage of fibrous callus formation, apparently faster than the monolithic PEEK-2.6 vol\% HA sample. After implanted for 3 months, the bone repair process for the PEEK-2.6 vol\% HA sample had entered the stage of fibrous callus formation with some connective tissues accompanied with inflammatory cells (Fig. 5b), while the tissues in contact with the FGM sample had developed a large quantity of connective tissues together with osteocytes and Haversian canals (Fig. 5d), suggesting that the new bone tissues around the implant sample became mature gradually. These observations confirm that HA has good bioactivity and can enhance bone growing, which is essential for bone formation [3]. As seen in Fig. 5, the bone repair process generally made progress with implantation time and HA contents in implants. Moreover, these results indicate that the bioactivity of the functionally graded composite materials can be greatly enhanced as compared with the 
(a)

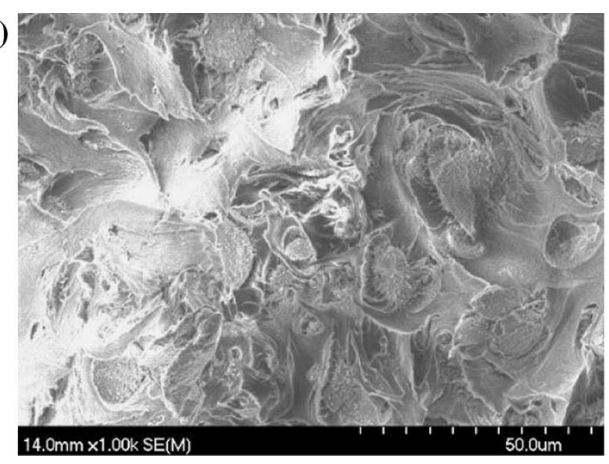

(b)

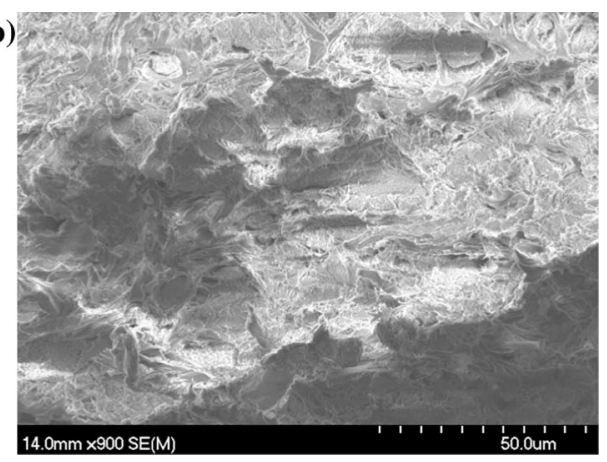

Fig. 4 The fractographs of PEEK composites with different HA contents: a 2.6 vol\% and b 8.7 vol\%

Fig. 5 Histological

micrographs of new bone tissues around the PEEK-HA and FGM implants: a PEEK2.6 vol\% HA for 1 month; b PEEK-2.6 vol\% HA for 3 months; c FGM for 1 month; and $\mathbf{d}$ FGM for 3 months; e autogenous bone for 1 month; f autogenous bone for 3 months (a)

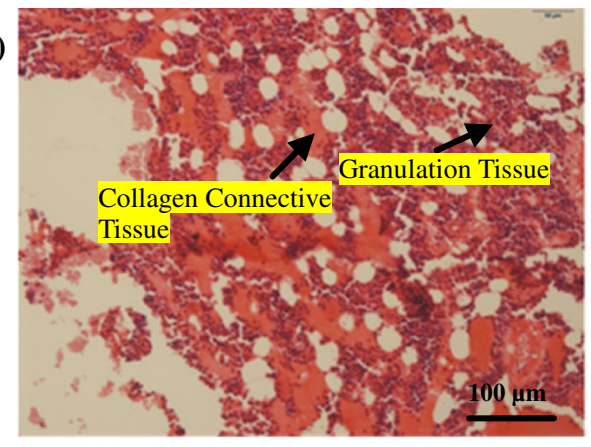

(c)

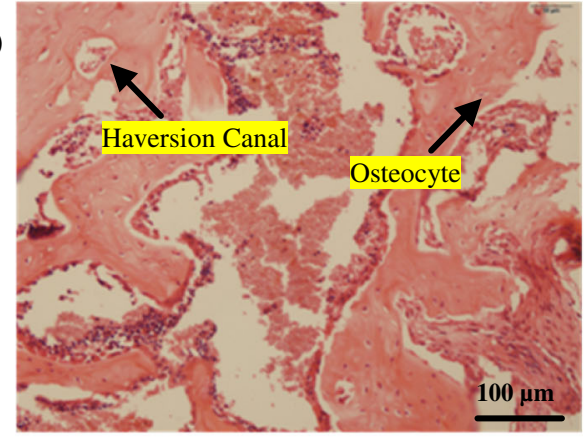

(e)

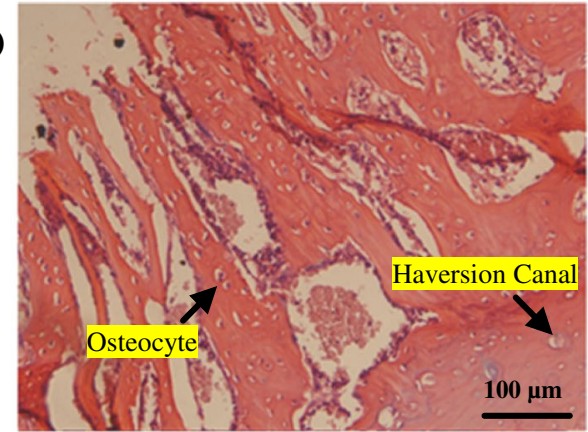

(b)

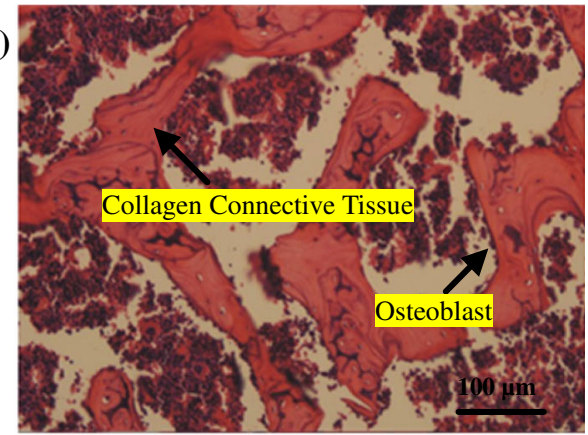

(d)

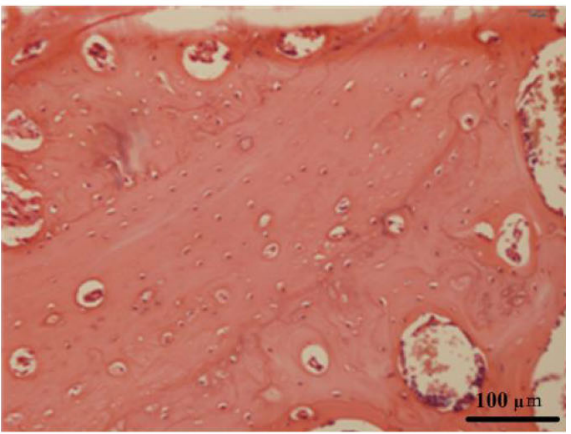

(f)

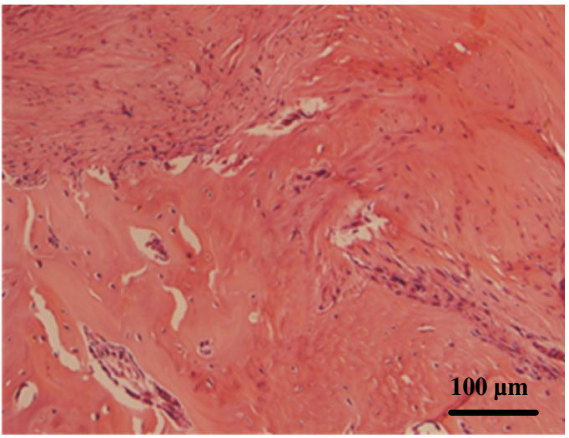

monolithic PEEK-2.6 vol\% HA composite materials. However, it was found that new bone tissues around the autogenous bone implant were more mature, obviously faster than FGM and PEEK-2.6 vol\% composite materials. There were a large amount of connective tissues around antogenous bone implant for 1 month implant, together with osteocytes and Haversian canals (Fig. 5e). After 3 months, the bone tissues with an orderly cell arrangement, together with osteocytes and Haversian canals, were observed (Fig. 5f), suggesting autogenous bone implant has better osteoconduction and bone regeneration capability. 
Table 1 The growth of new born around the implant samples for 3 months

\begin{tabular}{llllll}
\hline Group & $\mathrm{N}$ & Cross section $\left(\mathrm{mm}^{2}\right)$ & The average thickness $(\mathrm{mm})$ & Maximum thickness $(\mathrm{mm})$ & Minimum thickness $(\mathrm{mm})$ \\
\hline PEEK-2.6 vol\% HA & 3 & $0.580 \pm 0.201$ & $0.088 \pm 0.033$ & $0.131 \pm 0.051$ & $0.044 \pm 0.015$ \\
FGM & 3 & $0.720 \pm 0.433$ & $0.111 \pm 0.053$ & $0.178 \pm 0.081$ & $0.045 \pm 0.024$ \\
Host bone & 3 & $3.016 \pm 0.343$ & $0.484 \pm 0.098$ & $0.752 \pm 0.178$ & $0.216 \pm 0.021$ \\
\hline
\end{tabular}

Table 1 shows the growth of the new born bone around the implants of the FGM, monolithic PEEK-2.6 vol\% HA composite as well as autogenous bone samples for 3 months by the analysis of Image-Pro Express $\mathrm{C}$. The group of host bone showed significant bone-growing over the other groups of $2.6 \mathrm{vol} \% \mathrm{HA} / \mathrm{PEEK}$ and FGM $(p<0.01)$, whereas FGM versus 2.6 vol\% HA/PEEK comparison reached statistical discrepancies $(p=0.046<0.05)$. As seen in Table 1, the average thickness and cross section of the new born bone around FGM implant were respectively about 126 and $124 \%$ of the monolithic PEEK-2.6 vol\% HA composite sample, due to higher HA content in implant accelerating new born bone growing, in accordance with histological observation of the above. And furthermore, the average thickness and cross section of bone tissue in contact with host bone sample were respectively about 436 and $420 \%$ of FGM implant, suggesting that autogenous bone implant has great advantage in osteoconduction and bone regeneration ability over others' implants, in accordance with histological observation.

The clinical implants for hard tissue must match with the properties of host bone in vivo. The FGM researches of the above show that implant materials can really realize the balance between mechanical properties and bioactivity by integral structure design. And the mechanical properties of the functionally graded composites may be modified to a certain degree to meet various application requirements by optimizing the configuration design, such as each layer composition, and the thickness and number of layers. However, the previous reports show the bioactivity unsurprisingly increases with HA content in composites [7]. And the mechanical strength and ductility of the composite materials decreased substantially with HA content increase [12]. Therefore, the design of FGM for replacing hard tissues, is firstly needed to maintain enough mechanical properties, consequently restricting HA contents in FGM. It is found that autogenous bone in vivo test had unsurpassable ability on osteoconduction and bone regeneration, over others' samples with different HA contents. Draenert et al. [21] had evaluated the biological effect of the growth factor [bone morphogenetic protein (BMP)] on human osteoblasts, which showed that sustained release of BMP on bone biomaterials had a stronger positive effect on biological scaffolds. Chitosan/nano-HA/collagen composite incorporating BMP was fabricated by
Feng et al. [22], successfully accelerating the healing of rat's cranial bone defects. In conclusion, the further research is much needed to incorporate BMP into FGM for promoting bone regeneration.

\section{Conclusions}

A functionally graded material of PEEK-HA with a symmetrical three-layer structure containing 8.7, 2.6 and 8.7 vol\% HA, respectively, is successfully fabricated by hot pressing. The FGM samples exhibit fracture without falling apart or delaminating during tensile loading, indicating a strong cohesion between the layers. The tensile strength of the FGM is $86.2 \mathrm{MPa}$, which is about the average value of the monolithic PEEK-2.6 vol\% HA and PEEK-8.7 vol\% HA composite samples. However, the fracture energy of the FGM is $56.5 \mathrm{~kJ} / \mathrm{m}^{2}$, which is about $520 \%$ of that of the monolithic composite sample with 8.7 vol\% HA. The new born bone grew on the FGM implant, apparently faster than the PEEK-2.6 vol\% HA implant, indicating the bioactivity increases with HA content. However, autogenous bone implant exhibits maturer bone characteristics over the FGM implant, suggesting that the autogenous bone implant has unsurpassable ability on bone regeneration. The balance between mechanical properties and bioactivity may be achieved by virtue of a proper design of FGM and introduce of BMP. Therefore, the PEEK-HA FGM could be a promising biomaterial for natural bone replacement in the future.

Acknowledgments This work was supported by the Key Program of the National Natural Science Foundation of China (Grant No. 61136001), the Specialized Research Fund for the Doctoral Program of Higher Education of China (Grant No. 20114408110002), and the Key Laboratory of New Lithium-ion Battery Cathode Materials in Shenzhen City.

Open Access This article is distributed under the terms of the Creative Commons Attribution License which permits any use, distribution, and reproduction in any medium, provided the original author(s) and the source are credited.

\section{References}

1. Abdal-hay A, Hwang M-G, Lim JK (2012) In vitro bioactivity of titanium implants coated with bicomponent hybrid biodegradable polymers. J Sol-Gel Sci Technol 3:756-764 
2. Zhang QW, Mochalin VN, Neitzel I, Hazeli K, Niu JJ, Kontsos A, Zhou JG, Lelkes PI, Gogotsi Y (2012) Mechanical properties and biomineralization of multifunctional nanodiamond-PLLA composites for bone tissue engineering. Biomaterials 20:5067-5075

3. Ghomi H, Fathi MH, Edris H (2011) Preparation of nanostructure hydroxyapatite scaffold for tissue engineering applications. J Sol-Gel Sci Technol 3:642-650

4. Wang M, Bonfield W (2001) Chemically coupled hydroxyapatite-polyethylene composites: structure and properties. Biomaterials 11:1311-1320

5. Bakar MSA, Cheang P, Khor KA (2003) Mechanical properties of injection molded hydroxyapatite-polyetheretherketone biocomposites. Compos Sci Technol 3-4:421-425

6. Wang L, Weng L, Song S, Sun Q (2010) Mechanical properties and microstructure of polyetheretherketone-hydroxyapatite nanocomposite materials. Mater Lett 20:2201-2204

7. Bakar MSA, Cheng MHW, Tang SM, Yu SC, Liao K, Tan CT, Khor KA, Cheang P (2003) Tensile properties, tension-tension fatigue and biological response of polyetheretherketonehydroxyapatite composites for load-bearing orthopedic implants. Biomaterials 13:2245-2250

8. Mishina H, Inumaru Y, KaitokU K (2008) Fabrication of ZrO2/ AISI316L functionally graded materials for joint prostheses. Mater Sci Eng 1-2:141-147

9. Pei X, Wang J, Wan Q, Kang L, Xiao M, Bao H (2011) Functionally graded carbon nanotubes/hydroxyapatite composite coating by laser cladding. Surf Coat Technol 19:4380-4387

10. Rabiei A, Blalock T, Thomas B, Cuomo J, Yang Y, Ong J (2007) Microstructure, mechanical properties, and biological response to functionally graded ha coatings. Mater Sci Eng 3:529-533

11. Ma R,Weng L,Bao X,Ni Z,Song S Cai W (2012) Characterization of in situ synthesized hydroxyapatite/polyetheretherketone composite materials. Mater Lett 71:117-119

12. Wang L, Weng L, Song S, Zhang Z, Tian S, Ma R (2011) Characterization of polyetheretherketone-hydroxyapatite nanocomposite materials. Mater Sci Eng 10-11:3689-3696

13. Wong KL, Wong CT, Liu WC, Pan HB, Fong MK, Lam WM, Cheung WL, Tang WM, Chiu KY, Luk KDK, Lu WW (2009)
Mechanical properties and in vitro response of strontium-containing hydroxyapatite/polyetheretherketone composites. Biomaterials 23-24:3810-3817

14. Dhanalakshmi CP, Vijayalakshmi L, Narayanan V (2012) In situ preparation and characterization of nano carbonated hydroxyapatite/poly(4-vinyl pyridine-co-styrene) composite and its biomedical application. J Biomater Tissue Eng 4:314-321

15. Lim YM, Hwang KS, Park YJ (2001) Sol-gel derived functionally graded TiO2/HAP films on Ti-6Al-4V implants. J Sol-Gel Sci Technol 1-2:123-128

16. Afzal MAF, Kesarwani P, Reddy KM, Kalmodia S, Basu B, Balani K (2012) Functionally graded hydroxyapatite-aluminazirconia biocomposite: synergy of toughness and biocompatibility. Mater Sci Eng 5:1164-1173

17. Ma R, Weng L, Fang L, Luo Z, Song S (2012) Structure and mechanical performance of in situ synthesized hydroxyapatite/ polyetheretherketone nanocomposite materials. J Sol-Gel Sci Technol 62:52-56

18. Odegard GM, Clancy TC, Gates TS (2005) Modeling of the mechanical properties of nanoparticle/polymer composites. Polymer 2:553-562

19. Fan JP, Tsui CP, Tang CY, Chow CL (2004) Influence of interphase layer on the overall elasto-plastic behaviors of HA/PEEK biocomposite. Biomaterials 23:5363-5373

20. Kutvonen A,Rossi G Ala-Nissila T (2012) Correlations between mechanical, structural, and dynamical properties of polymer nanocomposites. Phys Rev E 4:041803-1-041803-6

21. Draenert FG, Nonnenmacher AL, Kammerer PW, Goldschmitt J, Wagner W (2013) Bmp-2 and bfgf release and in vitro effect on human osteoblasts after adsorption to bone grafts and biomaterials. Clin Oral Implant Res 7:750-757

22. Feng B, Hu DX, Zhang YD (2012) Accelerated bone regeneration by chitosan/nanometer hydroxyapatite/collagen composite incorporating bmp-7 mimetic peptide. J Hard Tissue Biol 4:481-487 